\title{
CSEB Student Conference 2012 abstract winners
}

\section{Preface}

Chronic Diseases and Injuries in Canada (CDIC) was pleased to manage, once again, the student abstract contest for the Canadian Society for Epidemiology and Biostatistics (CSEB) Student Conference, which was held at the University of Saskatchewan in May 2012. An editorial panel from the Public Health Agency of Canada judged 42 abstract submissions and selected the top 7 to be published in this issue of the journal.

The editorial panel consisted of the following members:

- Howard Morrison, PhD, Editor-inChief, CDIC

- Kenneth Johnson, PhD, Senior Epidemiologist

- Ania Syrowatka, MSc, Epidemiologist

- Michelle Tracy, MA, Managing Editor, CDIC

The selected abstracts were judged on their originality, clarity, scientific and technical excellence, and potential impact. The following questions helped guide the judges:

1. Is it relevant to chronic diseases and/ or injuries?

2. Are the data Canadian, or if not, do the authors place the issue in the context of Canada?

3. Does the study have national relevance? Local studies are of interest only to the extent that they provide sufficient details to allow them to be useful to other, non-local researchers.

4. Does the study address a significant public health issue?

5. Is the study scientifically rigorous?

6. Are the methods and/or results novel, or is it a significant improvement on previous studies of the same issue?

7. Can you imagine any reasonable circumstance where a different author would reference this study?
Since 2009, CDIC has collaborated with CSEB to foster publishing opportunities for students. CDIC is proud to collaborate with CSEB again this year and to encourage students in their publishing efforts. On behalf of the CDIC editorial team, I would like to thank all students who submitted their abstracts and to congratulate the winners. Having one's abstract published in a peer-reviewed journal is a good place to start in science publishing! We look forward to seeing future submissions of full research articles.

\section{Michelle Tracy, MA}

Managing Editor, Chronic Diseases and Injuries in Canada 


\title{
The effect of gender and geography on the self-rated mental health of single parents
}

\author{
A. Banerjee, MBBS (1); B. Janzen, PhD (1)
}

Introduction: Single parents, one of the most socio-economically vulnerable groups in Canada, consistently report poorer mental health compared with cohabiting parents. However, most of the studies examining the mental health of single parents typically fail to consider whether they live in urban or rural areas, or else focus exclusively on urban dwellers. This is despite that 1) a growing body of research points to place (e.g. rurality) as a determinant of health and 2) in 2006, just over $13 \%$ of families residing in rural Canada were headed by a single parent. In addition, little is known about the mental health of single fathers despite their increasing numbers in Canada.

Objective: To determine if the mental health of single parents varies by gender and/or rurality and what factors (e.g. economic, social) contribute to variations in mental health by gender and/or rurality.

Methods: From Statistics Canada's 2007/ 2008 Canadian Community Health Survey (Master file), we selected for analysis a subsample of 18- to 64-year-old single parents with at least one child aged under
25 years living with them. The dependent variable was self-rated mental health, and the primary independent variables were sex and residence, the latter based on Statistics Canada's metropolitan influenced zone (MIZ) classification. Covariates included age, employment status, household income, home ownership, food security and sense of belonging to a community. A series of univariate, bivariate and multivariable logistic regression analyses were conducted to answer the research questions. Sampling weights and a bootstrap variance estimation program were used to address the complex sampling strategy.

Results: The sample (weighted) consisted of 1024856 single parents. Single mothers made up $81 \%$ of the sample, and the majority of single parents (86\%) lived in urban areas. Overall, $9.3 \%$ of single mothers and $7.0 \%$ of single fathers reported fair/poor mental health. The proportion of single fathers with fair/poor mental health was $6.7 \%$ in Census Metropolitan Areas/ Census Agglomerations (CMAs/CAs), $11.0 \%$ in strong/moderate MIZ and $4.6 \%$ in weak/no MIZ. Among single mothers, the proportion with fair/poor mental health was $9.5 \%$ in $\mathrm{CMA} / \mathrm{CA}, 7.9 \%$ in strong/ moderate MIZ and $8.2 \%$ in weak/no MIZ. Inspection of the preliminary results are suggestive of variation in self-rated mental health and access to economic/social resources (e.g. employment, food security, sense of belonging) according to gender and/or degree of rurality; however, additional analyses applying the appropriate variance estimation techniques are required to determine whether these differences are statistically significant. In addition, multiple logistic regression needs to be conducted to determine if any observed gender/residence differences in single parents' mental health remain statistically significant after adjustment for key covariates.

Conclusion: The results of the study enhance understanding of the diverse experiences of Canadian single parents and inform the development of more targeted policies directed at improving their mental health.

Keywords: health policy, social policy, mental health, social epidemiology, behavioural epidemiology, Canadian Community Health Survey 


\title{
Sitting, screen time and suicide: the relationship between sedentary activity and suicide ideation in Canadian adolescents and young adults
}

\author{
M. A. Bélair, BSSc (2); I. Colman, PhD (2)
}

Introduction: Suicide is the second leading cause of death among 15- to 24-yearolds in Canada. At $21.4 \%$, suicide rates among adolescents have remained constant despite declining rates in other developed countries. More than $50 \%$ of adolescents who commit suicide have a major depressive disorder.

Objective: To investigate whether a link exists between sedentary activity and suicide ideation in Canadians aged 15 to 24 years.

Methods: Using an initial sample of 8356 from the Canadian Community Health Survey (CCHS), Cycle 4 (2007/2008), 7914 adolescents and young adults aged 15 to 24 years were included in the analysis. We conducted Breslow-Day tests for effect modification to determine the need for stratification and multivariate logistic regression analysis to assess the relationship between sedentary activity and lifetime suicide ideation. Sedentary activity was classified into three categories: 0 to 15,15 to 34 and 35 plus hours per week.
Results: Those who were sedentary 15 to $34 \mathrm{~h} / \mathrm{wk}$ had odds ratio (OR) of lifetime suicidal ideation 1.18 times higher $(95 \%$ confidence interval [CI]: 0.99-1.41) than those who were sedentary 0 to $15 \mathrm{~h} / \mathrm{wk}$, while those who were sedentary $35+\mathrm{h} /$ wk had OR 1.41 times higher (95\% CI: 1.15-1.74) than those in the least sedentary group. When controlling for sex, age, self-perceived health, self-perceived mental health and body mass index (BMI), as well as modelling an interaction between sex and selfperceived health and between sex and BMI, the relationship between suicide ideation and sedentary activity for youth and young adults in the $35+\mathrm{h} / \mathrm{wk}$ exposure category remained significant with an adjusted OR of 1.33 (95\% CI: 1.06-1.68), whereas that for those in the 15 to $34 \mathrm{~h} / \mathrm{wk}$ exposure category was non-significant at 1.11 (95\% CI: 0.921.35). To interpret the interaction terms, we explored sex-stratified models. For males reporting poor/fair self-perceived health, OR of lifetime suicidal ideation was 1.26 (95\% CI: $0.82-1.26)$ whereas for females OR was 2.33 (95\% CI: 1.683.23) compared with the reference (good/very good/excellent self-perceived health). A 10-unit increase in BMI decreased odds of lifetime suicide ideation for males by 0.97 ( $95 \%$ CI: 0.731.28), whereas it increased odds of lifetime suicide ideation by 1.58 times (95\% CI: 1.29-1.92) for females.

Conclusion: A relationship exists between sedentary activity levels and lifetime suicide ideation among youth and young adults with 35 or more hours of sedentary activity per week. This is of concern as a greater proportion of adolescents and young adults spends more time being sedentary. However, the cross-sectional nature of the CCHS does not permit us to comment on the direction of this relationship. Further research using longitudinal data is recommended.

Keywords: mental health, Canadian Community Health Survey, depression, suicide 


\title{
Health status and service use among homeless individuals with mental illness: consistency between self-report and administrative health records in the At Home/Chez Soi Multi-site Trial
}

\author{
A. Hinds, MSc (3); J. Distasio, PhD (4); P. J. Martens, PhD (3, 5); M. Smith, MSc (5)
}

Introduction: Homeless individuals with poor health use health care services frequently.

Objective: To examine health status, health care and prescription drug use among mentally ill, homeless individuals and compare self-report and administrative data claims to estimate the degree of agreement between the two sources.

Methods: Baseline survey data from 100 participants of the Winnipeg site of the Mental Health Commission of Canada's At Home / Chez Soi research project were linked to de-identified administrative health records stored in the Repository at the Manitoba Centre for Health Policy. We analyzed demographic characteristics, homelessness histories and health service use as well as disease status for asthma, hypertension, arthritis and diabetes (using previously validated definitions). Participants were similarly classified using their survey responses. The degree of agreement between the two data sets was evaluated using cross tabulations and the kappa $[\kappa]$ statistic.

Results: There was $100 \%$ linkage of surveyed homeless people with the Repository data. In one year, $97 \%$ of participants had at least one ambulatory physician visit, with an age- and sexadjusted rate of 14.82 per person-years (overall Manitoba rate $=4.99$ per personyears); 34\% were hospitalized (adjusted hospital separation rate $=491$ per thousand person-years versus the Manitoba rate of 137 per thousand person-years); and $95 \%$ filled at least one prescription with $65 \%$ of drugs targeting the nervous system (the majority were psycholeptics). The degree of agreement between the disease-related data sources ranged from poor $(\kappa=0.27)$ for arthritis to moderate ( $\kappa=0.57)$ for hypertension. Individuals were more likely to be classified as having one of the four selected conditions based on the administrative data than on the survey data.

Conclusion: Compared to the general population, homeless participants had high health service use and high prescription drug use. There was poor to moderate agreement between the two data sources with regard to disease detection. Researchers studying homeless persons with mental illness should consider using multiple data sources to estimate disease prevalence and health service use.

Keywords: mental health, epidemiological methods, health care services use, administrative health records, homelessness

\footnotetext{
Author references:

3. Department of Community Health Sciences, University of Manitoba, Winnipeg, Manitoba, Canada

4. Institute of Urban Studies, University of Winnipeg, Winnipeg, Manitoba, Canada

5. Manitoba Centre for Health Policy, University of Manitoba, Winnipeg, Manitoba, Canada

Correspondence: Aynslie Hinds; Email: umhinds0@cc.umanitoba.ca
} 


\title{
Modelling costs of episodes of care for exacerbations of chronic obstructive pulmonary disease
}

\author{
J. P. Kuwornu, MSc (6); L. M. Lix, PhD (6, 7); J. M. Quail, PhD (6, 7); E. Wang, MSc (7); M. Osman, MA (7)
}

Introduction: An episode of care (EoC) is the cluster of health care services associated with an acute or chronic condition. EoCs are used to examine variations in cost and use of different treatment pathways. Predictive models of EoC utilization and costs can be used to identify the pathways that result in optimal treatment outcomes. However, a major issue associated with making valid and accurate predictions is the selection of an appropriate statistical model.

Objective: To compare different statistical models for predicting EoC costs for exacerbations of chronic obstructive pulmonary disease (COPD).

Methods: The study data included hospital separations, physician billing claims, prescription drug records and population registration files from Saskatchewan. The study cohort was made up of individuals aged 35 years and older with a COPD diagnosis in hospital or physician claims. We identified EoCs initiated by a hospitalization with a primary diagnosis of COPD from 2000/2001 to 2009/2010, and calculated total hospital, physician and drug costs for each EoC, adjusting for inflation. We compared marginal generalized estimating equation (GEE) and random effects models with a gamma or negative binomial distribution for mean EoC cost and quantile regression model for median EoC costs. Covariates included demographic, socio-economic and disease-related variables.

Results: From the study cohort ( $\mathrm{n}=41$ 848), we identified 20999 EoCs for COPD exacerbations initiated by hospitalisation. Average age of those who had COPD EoCs was 71 (standard deviation [SD]: 12) years, and $53 \%$ were male. Total costs were highly skewed. The median total cost was $\$ 4,506$, while the mean (SD) was $\$ 7,968(\$ 13,354)$. The median was higher for episodes $(n=2400)$ in which the individual died $(\$ 8,380)$ than not die $(\mathrm{n}=$ 18 599; \$4,400). The random effects and GEE models failed to converge for the gamma distribution. The model with a negative binomial distribution fit the data well based on the deviance statistic. All covariates in this model were statistically significant except for sex $(p=.8179)$ and age $(p=.0610)$. The quantile regression model also converged; only the Charlson comorbidity score was not statistically significant $(p=.5791)$.

Conclusion: Quantile regression and marginal models with negative binomial distribution appear to be valid approaches to addressing the small proportion of individuals with high EoC costs for COPD. The models produced different results about the significance of the covariates. The choice of models will influence the patient characteristics associated with health care costs and treatment trajectories, and could lead to different conclusions about optimal treatment pathways for COPD patients.

Keywords: longitudinal analysis, epidemiological methods, respiratory epidemiology 


\title{
Characteristics associated with increased pain and low functional recovery three to five years following total knee arthroplasty
}

\author{
J. E. Mollins, MSc (8); C. A. Jones, PhD (9, 10); M. Clark, MD (11); L. Beaupre, PhD (9, 10)
}

Introduction: The incidence of total knee arthroplasty (TKA) performed in Canada is steadily increasing; however, $9 \%$ to $19 \%$ of all TKA patients experience little or no improvement in physical function and pain relief postoperatively. A lack of consensus exists about the factors associated with these poor outcomes. Determining baseline characteristics and demographics associated with increased pain and negated postoperative functional status could assist in identifying patients who are less likely to benefit from this operation. If these factors are modifiable, they could be addressed before TKA to improve postoperative outcome; if not, patients could be given realistic postoperative expectations.

Objective: To identify modifiable and nonmodifiable baseline patient demographics associated with poor pain and physical function scores on the Western Ontario McMaster Osteoarthritis Index (WOMAC) at 3 to 5 years following TKA.

Methods: This was a secondary analysis of prospectively collected data from the Alberta Arthroplasty Study, a large randomized clinical trial. We performed initial descriptive analyses and compared baseline scores between responders and non-responders as well as univariate linear regression for the following independent variables: age, gender, group allocation, body mass index (BMI), categorical comorbidities ( $\leq 2$ or $\geq 3$ conditions), presence of back pain, diabetes status, presence of lung disease, smoking status, baseline Medical Outcomes Study 36-item Short Form (SF-36) mental health (MH) scores, baseline WOMAC physical function scores and baseline WOMAC pain scores. This initial model building step was performed twice: once with WOMAC pain scores and once with WOMAC function scores as the dependent variable. A multivariate regression was then developed using purposeful selection techniques. Final stability of the model was assessed using forward and backward stepwise regression methods to determine agreement among significant variables; variance inflation factors were calculated to test for collinearity.

Results: A total of 388 patients consented to further evaluation 3 to 5 years after TKA. We observed significant improvements in both WOMAC pain and function scores. In the multivariate analyses, older age, presence of back pain, and overweight or obesity were indicators of both worse pain levels and inferior functional status. Better preoperative WOMAC pain and SF-36 MH scores were associated with improved postoperative pain levels. Higher WOMAC function and SF-36 MH scores at baseline were predictive of better functional outcomes post-TKA. Coefficients of determination $\left(R^{2}\right)$ were 0.15 for the pain model and 0.19 for the function model.

Conclusion: Older age is associated with poorer pain outcomes several years postTKA; however, older individuals experienced changes in pain similar to that of younger patients. Thus, older age should not be a limiting factor when considering candidates for TKA. Increased BMI was also a significant predictor of long-term pain and function scores. Interventions to manage BMI and back pain should be considered preoperatively to maximize TKA outcomes. Based on the identified risk factors, patient expectations may be revised regarding outcomes. Low $R^{2}$ values indicate limited ability of the model to predict patient outcomes 3 to 5 years following surgery. Future research may consider including more psychosocial variables in medically based models when assessing TKA outcomes.

Keywords: clinical epidemiology, longitudinal analysis, aging

\footnotetext{
Author references:

8. Faculty of Rehabilitation Medicine, University of Alberta, Edmonton, Alberta, Canada

9. Department of Physical Therapy, University of Alberta, Edmonton, Alberta, Canada

10. Alberta Innovates - Health Solutions, Edmonton, Alberta, Canada

11. Department of Surgery, University of Alberta, Edmonton, Alberta, Canada

Correspondence: Juliana Mollins; Email: mollins@ualberta.ca
} 


\title{
Variable importance measures for non-normal data: an application to patient-reported outcomes on their health- related quality of life
}

\author{
T. T. Sajobi, PhD (12); B. M. Dansu, PhD (12); L. M. Lix, PhD (12)
}

Introduction: Health-related quality of life (HRQOL) measures are widely used in clinical trials to assess the effectiveness of new treatments across physical, psychological and social domains. Variable importance measures derived from descriptive discriminant analysis (DDA) and multivariate analysis of variance (MANOVA) procedures have been developed for evaluating the importance of domain for HRQOL data collected at a single time point. This includes standardized discriminant function coefficients, discriminant ratio coefficients, and F-to-remove statistics. However, these measures may result in inconsistent rank ordering of domains in HRQOL data characterized by non-normal distributions.

Objective: To develop and apply measures of relative importance derived from DDA and MANOVA procedures based on trimmed means and Winsorized covariances for evaluating domain importance in non-normal multivariate data.

Methods: DDA and MANOVA procedures that are insensitive (i.e. robust) to departures from multivariate normality assumption were developed by replacing the least squares estimates of means and covariances with trimmed means and Winsorized covariances, respectively. Variable importance measures derived from the coefficients of these robust DDA and MANOVA procedures were used to rank order variables in non-normal multivariate data. Variable importance measures based on least squares and robust estimators were illustrated using data from the Manitoba Inflammatory Bowel Disease Cohort Study, an on-going longitudinal cohort study that is investigating psychosocial predictors of health outcomes. Study participants with selfreported active $(n=265)$ and inactive ( $n=116$ ) disease were compared on the four domains of the disease-specific inflammatory bowel disease questionnaire (IBDQ) and the eight domains of the Medical Outcomes Study 36-item Short Form (SF-36) Questionnaire that measured the physical and mental aspects of participants' health and well-being.

Results: When measures of relative importance based on least squares estimators were used to evaluate domain importance, the IBDQ bowel symptom and the SF-36 general health domains were identified as the most important domains. In contrast, the IBDQ emotional health and the SF-36 general health domains were identified as the most important domains that discriminate between active and inactive disease groups when variable importance measures based on trimmed means and Winsorized covariances were used to evaluate domain importance. The rank ordering of the remaining domains varied across variable importance measures and estimation methods.

Conclusion: These relative importance measures can be used to choose a parsimonious subset of domains that best discriminate between groups in non-normal HRQOL data. Further research is needed to investigate the properties of these measures under a variety of data analytic conditions.

Keywords: biostatistics, population, public health 


\title{
Women's health care utilization among HIV-positive women on ART in British Columbia*
}

\author{
X. Wang, MPH (13); K. A. Salters, MPH (14); H. Wang, MSc (14); W. Zhang, MSc (14); N. Pick, MD (15, 16); \\ J. S. Montaner, MD (14, 15); R. S. Hogg, PhD (13, 14); A. Kaida, PhD (13)
}

*This abstract appears in a full version of the article with the following citation: Wang X, Salters KA, Zhang W, McCandless L, Money D, Pick N, Montaner JSG, Hogg RS, Kaida A. Women's Health Care Utilization among Harder-to-Reach HIV-Infected Women ever on Antiretroviral Therapy in British Columbia. AIDS Research and Treatment. 2012; doi:10.1155/2012/560361

Introduction: Women make up more than $50 \%$ of HIV-positive (HIV+) population globally and about $22 \%$ nationally. That there are experiences unique to women living with HIV has been well established. Compared with their HIV-negative counterparts, HIV+ women are more likely to have abnormal gynecological conditions and menopause-related problems such as osteoporosis. However, several studies have suggested that medical care specific to women was underutilized among HIV+ women despite that appropriate use of women's health care has been shown to reduce HIV-related burden of diseases.

Objective: To estimate the prevalence and covariates of women's health care utilization among HIV+ women who ever received antiretroviral therapy (ART) in British Columbia (BC).

Methods: The Longitudinal Investigations of Supportive and Ancillary Health Services (LISA) study is a study of people living with HIV who have ever received ART in various BC clinics. The crosssectional interview data on sociodemographic factors, supportive service use and quality of life were linked to the longitudinal HIV clinical data available through the provincial Drug Treatment Program. For this analysis, inclusion was restricted to LISA participants who identified as female. The outcome measure was current utilization of women's health care. The answers were dichotomous (yes vs. no) based on responses to the LISA survey question, "I have a physician who I see regularly for women's health care.” Independent covariates included demographic variables (age, ethnicity, health authority, rural/urban residency, marital status), sociodemographic variables (education, employment, income, housing stability, food security), psychosocial variables (stigma, perceived neighbourhood problems or cohesion, quality of life), substance-use variables (alcohol use, illicit drug use, drug injection), sexual health variables (sexual activity, condom use, sex trade history, pregnancy intention, number of births, history of sexually transmitted infections, abnormal Pap smear in last 6 months), mental health variables (symptoms of depression), and HIV clinical variables (ART status, CD4 cell count, plasma viral load, viral load suppression, duration of immunosuppression). Bivariate analyses and multivariable logistic regression analyses were conducted to identify factors associated with women's health care utilization.

Results: Of the 231 women participants, $77 \%$ regularly accessed women's health care. Median age was 41 years, 49\% reported Aboriginal ancestry, $72 \%$ had an annual income less than $\$ 15,000,62 \%$ had stable housing and $23 \%$ were food secure. In the multivariate analysis, factors associated with women's health care utilization included not living in Vancouver Island Health Authority (odds ratio $[\mathrm{OR}]=0.12$, $95 \%$ confidence interval [CI]: 0.04-0.37), no current illicit drug use (OR $=0.42,95 \%$ CI: 0.19-0.92), higher annual income (OR $=6.73$, 95\% CI: 1.85-24.54) and increased provider trust (QoL scale) (OR $=1.03$, 95\% CI: 1.00-1.05).

Conclusion: Despite a relatively high prevalence of women's health care utilization among HIV+ women on ART in BC, a health service gap persists along geographic and social axes. To effectively integrate women's health care into routine HIV care, programs and services need to be tailored to women's needs by addressing social and structural determinants of health.

Keywords: social epidemiology, behavioural epidemiology, women's health, health services research

\footnotetext{
Author references:

13. Faculty of Health Sciences, Simon Fraser University, Burnaby, British Columbia, Canada

14. BC Centre for Excellence in HIV/AIDS, Vancouver, British Columbia, Canada

15. Faculty of Medicine, University of British Columbia, Vancouver, British Columbia, Canada

16. Oak Tree Clinic, BC Women's Hospital and Health Centre, Vancouver, British Columbia, Canada

Correspondence: Xuetao Wang; Email: wangxtk@gmail.com
} 